\title{
MedienPädagogik
}

Zeitschrift für Theorie und Praxis der Medienbildung

www.medienpaed.com

ISSN 1424-3636

Themenheft Nr. 37: Medienpädagogik als Schlüsseldisziplin in einer mediatisierten Welt. Perspektiven aus Theorie, Empirie und Praxis Herausgegeben von Henrike Friedrichs-Liesenkötter, Lara Gerhardts, Anna-Maria Kamin und Sonja Kröger

\section{Jugend als Mythos - Mythen im Film}

\section{Ein Essay}

Renate Möller und Uwe Sander

\section{Zusammenfassung}

Jugend, das ist die These, soll als zentraler Mythos der Moderne angesehen werden. Dabei ist mit Jugend weniger das empirische Phänomen einer eigenständigen und intern ausdifferenzierten Lebensphase gemeint, sondern eher die Konstruktion einer idealen Generationeneinheit, die durch Opposition zur Gegenwart und nicht als Faktum Sinn und Identität vermitteln soll. Als Fiktion und Konstruktion braucht jeder Mythos, auch der der Jugend, ein Trägermedium. Geschichten eignen sich hierzu am besten, sei es in Form von mündlich tradierten Erzählungen, in Form von Romanen oder Filmen. Filme können die narrative und mythengenerierende Tradition der Literatur direkt aufgreifen. Sie bieten Projektionsflächen im eigentlichen, aber auch im übertragenen Sinn und können dem Mythos Jugend eine akustische und optische Form geben. Jugendmythen in Filmen benötigen nicht unbedingt künstlerische Qualität oder aufwendige Inhalte. Vielmehr kommt es darauf an, dass sich Jugendliche mit den Filmen identifizieren und sie als Sprungbrett in alternative Phantasiewelten nutzen können. 


\title{
Youth as Myth - Myths in Film. An Essay
}

\begin{abstract}
Youth, that is the thesis, is to be regarded as the central myth of modernity. Youth is not so much the empirical phenomenon of an independent or biological phase of life, but rather the construction of an ideal generational unit, which should convey meaning and identity through opposition to the present and not as an empirical fact. As fiction and construction, every myth, including that of youth, needs a carrier medium. Stories are best suited for this purpose, whether in the form of orally handed-down narratives, novels or films. Films and movies can directly take up the narrative and myth-generating tradition of literature. They offer projection screens in the actual but also in the figurative sense and can give the myth of youth an acoustic and optical form. Youth myths in films or movies do not necessarily require artistic quality or elaborate content. More important is that young people identify with the films and can use them as a springboard to alternative fantasy worlds.
\end{abstract}

\section{Mythos und Realität}

Der Porsche 550 Spyder, ein kleiner, flacher Rennwagen aus den 50er Jahren, hat nach heutigen Verhältnissen kaum Besonderes zu bieten. Sein VierZylinder-Motor mit $1.500 \mathrm{ccm}$ liefert ca. 110 bis 135 PS und beschleunigt die gut $500 \mathrm{~kg}$ Auto auf max. $240 \mathrm{~km} / \mathrm{h}$. Auf heutigen Autoausstellungen und Oldtimer-Auktionen allerdings strahlt dieses meist silberne Fahrzeug eine ungebrochene Faszination aus. Sein Mythos lebt vom Tod des 24-jährigen James Dean, der am 30. September 1955 in Kalifornien auf dem Highway 466 frontal durch die Wuchtigkeit eines entgegenkommenden Ford ins Jenseits befördert wird. Ohne zu bremsen (wie im Film!), und das heisst hier: in jugendlich-männlicher Coolness darauf vertrauend, dass andere sich zurücknehmen werden und nicht man selbst, stirbt ein Mensch - und eine Legende kann weiterleben. James Dean gilt fortan (wie sein damaliges Gefährt) als Prototyp grazil-aggressiver, unangepasster Jugendlichkeit. Allein das reichte schon. Drei Filme und der dazu passende Abgang verschaffen (eine Zeitlang) Ewigkeit und sind genug Stoff für einen Mythos. 
Viel mehr wäre vielleicht auch zuviel gewesen, droht jedem Mythos doch das Aus durch die profane Wirklichkeit, konkret beim Jugendmythos: das Erwachsenenalter. Eigentlich zwingt das klassische Forever-young die Idole der Jugend dazu, sich beizeiten zu verabschieden, damit ihr Mythos weiterleben kann.

Auf eines werden wir noch einige Male hinweisen. Mythen würden sich an zu viel Realismus zerreiben; sie brauchen die Formbarkeit des Fiktiven, des Konstruierbaren, des Phantasierbaren und müssen sich, um Kraft zu gewinnen, von störender Bodenhaftung frei halten. Mythen (und zwar ganz allgemein: Personen, Geschichten oder Ereignisse) heben nicht auf Realität ab, sondern stellen Gegenbilder, Fiktionen, Simulakra der Erfahrungswelt dar. Sie überspringen Ratio und Empirie und suchen mit Unwirklichkeit zu verzaubern. Nur so lassen sie sich als Projektionsflächen für Eskapismen, Wünsche und Sehnsüchte einsetzen. Mythen müssen geheimnisvoll, offen und träumbar sein, eben wie der Mythos James Dean.

Kommt die Realität ungebeten in die Quere, dann verweltlicht der Mythos und braucht zum weiteren Überleben neue Unbestimmtheit. Notwendig wurde das z.B. bei Greta Garbo, die erst durch das Management von Harry Edington zur 〈Garbo〉 und zur 〈Göttlichen〉 wurde, indem Interviews mit der Presse unterbunden wurden und sich eine wissbegierige Öffentlichkeit ihr Garbo-Bild aus Melancholie und kühlem Charme selbst zusammenbasteln musste. Aus den Filmbildern von Unnahbarkeit und körperloser Leidenschaft liessen sich dann - je nach Gusto - konkrete Phantasmen und Kopfbilder durch das Publikum formen. So gewährt der Mythos die Freiheit der Indienstnahme. Allerdings nicht unterwürfig und nicht ohne Macht, nutzt der Mythos doch nach Roland Barthes (2010) unser zentrales Reservoir der Wirklichkeit, die Sprache, parasitär, okkupiert sie und schafft eine imaginäre Realität, die sich aufdrängen kann und zur unhinterfragten Welt konvertiert.

\section{Mythos Jugend}

Es ist schon angeklungen. Wenn Jugend als Mythos aufgefasst wird, handelt es sich bei dem Bezeichneten nicht um Realität, also nicht um die empirische Faktizität gegenwärtiger Jugendlicher mit ihren spezifischen 
Lebensmustern, sondern um Jugend als Stilisierung, Konstruktion und Fiktion. Greifen wir den Mythos Langemarck auf, so wird dieses deutlich. Im Laufe der Verstösse deutscher Truppen am 10. November 1914 mit ihren nur unzureichend ausgebildeten Reservekorps junger Kriegsfreiwilliger kamen über 2.000 junge Soldaten bei dem Versuch um, eine Hügelkette in der Nähe der Ortschaft Langemarck einzunehmen. Real wurden den heranstürmenden Schülern und Studenten durch die Maschinensalven der Gegner die Eingeweide aus dem Leibe geschossen, und die Schlacht artete zu einem chaotischen Schlachten aus. Ex post entstand daraus der Mythos von Langemarck, indem der offizielle Heeresbericht von Heldentod und Opferbereitschaft sprach und die Jugendlichen heroisch mit dem Deutschlandlied auf den Lippen für Nation und Vaterland sterben liess. Über Weimar hinaus konnte sich dieser Mythos anscheinend unbeschadeter halten als die kollektiv erlebten Erfahrungen der Grauen des Ersten Weltkriegs. Der Mythos vom Krieg tritt hier an die Stelle des Krieges und kann nur wenige Jahrzehnte später eine erneute Begeisterung auslösen.

Jeder Mythos besitzt diesen fiktiven Charakter und zeichnet sich aus durch eine oppositionelle und idealisierende Haltung gegen die Realität: So hat der Mythos eine negative Seite, die sich als Kritik auf einen aktuellen Zustand, also etwas Existentes, bezieht; und eine positive Seite, die als Widerpart zum Bestehenden eine ideale Gegenwelt aufbaut. Die negative Seite des Mythos Jugend wird durch die Erwachsenenwelt, im Prinzip die gesamte Gesellschaft mit ihren Zwängen, sowie durch das reale Jugendleben markiert; die positive Seite transzendiert ein Jugendideal, das sich Jugendlichen und Erwachsenen gleichermassen als Identifikationsfolie anbietet. Übernehmen Jugendliche einen Jugendmythos als den ihren, d.h. anerkennen sie ihr wahres bzw. erwünschtes Sein im jeweiligen Jugendmythos, gewinnt der Mythos eine eigentümliche Realität. Jugend als empirischer Faktor, obgleich real keineswegs dem Mythos Jugend entsprechend, wird dann mit dem Mythos Jugend in eins gesetzt. Der Jugendmythos bemächtigt sich parasitär der Realität und konvertiert zur kollektiv geteilten Fiktion sozialer Wirklichkeit. In diesem Übergang von der Mythenkonstruktion zur Identifikation mit dem Mythos lassen sich die Determinationen umkehren. Jugendmythen sind nun keine irisierenden Irrlichter mehr, aus Wünschen und Sehnsüchten von realen Jugendlichen oder Erwachsenen 
geboren. Vielmehr gebiert der Mythos nun autark die Sehnsucht nach sich selbst. Jugendliche (und wahrscheinlich sogar noch stärker Erwachsene) wollen so jugendlich sein wie die Jugend (als Mythos) ist.

Hier drängt sich natürlich sofort das Konzept der Identität als Assoziation auf, ist doch die reflektierende Selbstausleuchtung, ob man tatsächlich man selbst ist, nichts anderes als die Irritation zwischen der Differenz des realen Seins (so wie man wirklich ist) und des gewähnten Seins (so wie man glaubt, dass man eigentlich sei - wenn man nicht so wäre, wie man wirklich ist). Wir wissen mittlerweile sehr gut: Seit den Subjektphantasien der Moderne werden immer mehr Menschen von der Sorge umtrieben, nicht so zu sein, wie sie eigentlich sind. Und man weiss auch, dass gerade Medienvorbilder viel Verwirrung ins Identitäre bringen können, weil sie die Phantasie, wie man wirklich ist (aber eben gegenwärtig nicht ist), auch bei ansonsten eher phantasielosen Menschen anzuregen vermögen. Es ist demnach zu vermuten, dass Medien (hier insbesondere Filme) nicht nur fraglos hingenommene Identitäten aufschrecken, sondern auch komplexere Fiktionen (hier: Jugendmythen) provozieren können.

\section{Genesis des Jugendmythos}

Ich bin ein deutscher Jüngling (Frank 1989, 96) (Matthias Claudius, 1740-1815)

«Ich bin ein deutscher Jüngling

Mein Haar ist kraus, breit meine Brust

Mein Vater war ein edler Mann

ich bin es auch

Wenn mein Aug Unrecht siehet

sträubt sich mein krauses Haar empor

Und meine Hand schwellt auf und zuckt

und greift ans Schwert.»

Aus der historischen Rekonstruktion des sozialen Phänomens Jugend wissen wir, dass die Genese der Vorstellung eines Moratoriums zwischen Kindheit und Erwachsenenalter mit seinen idealisierten Charakteristika mit den Beginn der Moderne zusammenfällt (Sander 2000). 
Als Pädagogen und Literaten den Typus des romantisch verklärten Jünglings (s.o.) entwarfen, veränderten sich in Deutschland bzw. Europa die spätständischen Gesellschaftsstrukturen in Richtung funktionaler Differenzierung. Traditionelle Lebensgemeinschaften, sozialkulturelle Milieus und lokale Identitäten zerbrachen langsam und an ihre Stelle traten nach und nach ausdifferenzierte Gesellschaftsbereiche und funktionalisierte Lebensbezüge. Innerhalb dieses gesellschaftlichen Transformationsprozesses verloren nicht nur die traditionellen und vergemeinschaftenden Interdependenzverhältnisse an Bedeutung, auch die grossen Erzählungen und Weltbilder, also die traditionellen Markierungen von Synthesis und Einheit, fallen der analytischen Moderne zum Opfer.

Eine bislang statische gesellschaftliche Ordnung gerät in Bewegung, und Zukunft wandelt sich damit zu einer ambivalenten, schwer abzuschätzenden Grösse. Das erzeugt vielerlei Versuche der Sinnsicherung als Vergangenheitsorientierung (z.B. Romantik) und Absicherung des Bewährten (z.B. restaurative Bestrebungen), aber wenig innovative Utopien. Jugend war eine dieser innovativen Utopien als Option auf eine «bessere` Zukunft. Kennzeichnend für diese Visionen war ihre ausdrückliche Opposition zur Realität. Sie bezogen sich vornehmlich negativ auf die Repräsentanten einer politischen, bürokratischen und industriellen Moderne und suchten antithetisch Heil im Transzendenten, Idealen, Zukünftigen. Nach dem Ende des Kaiserreichs 1918 etwa wurde der bis dahin schon in einem Amalgam aus reformpädagogischen Traditionen, Lebensphilosophie, deutschem Idealismus, antimoderner Kulturkritik und Jugendbewegung vorbereitete Jugendmythos als Hoffnungsträger einer neuen Gesellschaft instrumentalisiert, wie dann noch einmal in Form eines nationalsozialistischen Plagiats nach 1933. Der Mythos Jugend war in dieser Zeit zwar längst geboren, erhielt allerdings immer mehr und immer verschiedene Konturen, nicht zuletzt durch die Jugendforschung und -theorie.

Worauf gründet nun dieser Jugendmythos? Paradoxer- oder vielleicht auch verständlicherweise wird in der Entstehungsphase des Jugendmythos der Begriff des Mythos allgemein als Widerspruch zur neuen analytischen Vernunft wie ein Kampfbegriff eben gegen die immer augenscheinlicheren Modernisierungstendenzen gebraucht. 
«Mythen dienen, den Bestand und die Verfassung einer Gesellschaft aus einem obersten Wert zu beglaubigen. Man könnte das die kommunikative Funktion des Mythos nennen, weil sie auf das Verständigtsein der Gesellschaftsteilnehmer untereinander und auf die Einträchtigkeit ihrer Wertüberzeugungen abzielt» (Frank 1989, 96).

Mythen sollen demnach das Verlorengegangene an traditioneller Einheit im Prozess der sozialen Differenzierung wieder herstellen. Ein historisch kraftvoller und überdauernder Mythos entsteht aus diesem Zusammenhang mit den ehemals revolutionären, nun aber konservativ bis reaktionär gewendeten Einheitskonzepten von Volk und Nation. Volk und Nation, so hinterlässt eine historische Rückschau den Eindruck, sollten als ideologische Verklammerung die komplexen sozialen, politischen und ökonomischen Ausdifferenzierungen der Moderne ausgleichen und als Religionsersatz dienen. Im ausgehenden 19. und beginnenden 20. Jahrhundert werden diese völkischen Einheitsmythen dann noch biologistisch mit Blut und Rasse angereichert und erfahren nach dem Ende des zweiten Jahrtausends bis heute eine beängstigende Renaissance.

Jugend, und das ist hier die These, soll als zentraler Mythos der Moderne angesehen werden. Dabei ist mit Jugend weniger das empirische Phänomen einer eigenständigen und intern ausdifferenzierten Lebensphase gemeint, sondern eher die Konstruktion einer idealen Generationeneinheit, die durch ideale Opposition zur Gegenwart und nicht als Faktum Sinn und Identität vermitteln soll. Allgemein stellt sich natürlich die Frage, ob eine Gesellschaft, der die gemeinschaftsstiftende Kraft eines einheitlichen Weltsinns auf lebenspraktischer Ebene abhanden gekommen ist, überhaupt im Mythos Ersatz finden kann. Dieses sind auch die charakteristischen Fragestellungen einer Gesellschaftskritik, in der der Bindungsverlust moderner Gesellschaften als Sinnkrise interpretiert wird. Die tragenden, letztlich für alle geltenden Verbindlichkeiten einer traditionellen, durch Religion und Mythos fixierten Sinngebung des Lebens seien aufgezehrt, so heisst es in der kulturkritischen Skepsis, aus der auch der Mythos Jugend entspringt. Für die klassischen Institutionen und Ordnungen der Gesellschaft bedeutet dieses keineswegs einen Bestandsverlust, jedoch geraten Politik, Erziehungssystem, Kirche, Ehe etc. in Legitimationskrisen, 
da eine unausgesprochene Rechtfertigung dieser Institutionen von sich aus nicht mehr gegeben ist. Die Gesellschaft mit ihren Teilsystemen funktioniert zwar weiter, für die in diesen institutionellen Rahmenbedingungen vergesellschafteten Einzelnen wird jedoch die Legitimität der Ordnung tendenziell zur leeren Hülse. Legitimität wird ersetzt durch die wenig verlässliche und labile normative Kraft des Faktischen. Was ist, besteht zwar, allerdings ohne Zusammenhang und Zusammenhalt. Die Perspektive der Bindungskrise als Sinnkrise bezieht sich demnach nicht auf den Geltungsverlust einzelner gesellschaftlicher Ordnungskriterien, sondern befürchtet die Atomisierung einer gesellschaftlichen Ordnung durch das Fehlen einer übergeordneten Kraft (z.B. eines Mythos).

Die verantwortliche Instanz in den Kritiken der Sinnkrise ist hinlänglich bekannt. Es ist die Aufklärung bzw. die Moderne selbst, die dem sich emanzipierenden Individuum und der sich emanzipierenden Gesellschaft ihre sinnhaften Grundlagen geraubt hat. Vernunft, Technik, Modernität usw. haben den Mythos, den umgreifenden Sinn, besiegt, aber die vereinheitlichende Funktion des Mythos nicht kompensieren können.

Also gilt anscheinend die Perpetuierung: Der Mythos ist tot, es lebe der neue Mythos! Und mit dem Konzept der Jugend kann gerade immer dann ein neuer Mythos inauguriert werden, wenn, wie Nietzsche es ausdrückte, mit dem aufziehenden Nihilismus «der unheimlichste aller Gäste» vor der Tür steht (Nietzsche 1969, 424).

Was zeichnet nun genauer den Mythos Jugend aus? Diese Frage kann nicht inhaltlich-sachlich geklärt werden, sondern nur strukturell, weil der Mythos Jugend als Formkonzept inhaltlich und historisch variabel, jedoch in seinen Grundbedingungen durch feste Standards ausgezeichnet ist. Jugend als Mythos konnte sich anscheinend als universelle Projektionsfläche für moderne Gesellschaft etablieren, in denen es zur Mode wurde, die Gegenwart mit ihren Beschränkungen zu transzendieren und zu überwinden. Diese Hoffnungsfolie kann beliebig politisch ausgeschlachtet werden und eignet sich sowohl für gesellschaftspolitische Phantasien (Jugend als Fackelträger der Neuen Zeit) wie auch für den individuellen Kampf gegen Alter, Gesetztheit und Tod (Jugendlichkeit als kultureller Habitus von Jugendlichen und Erwachsenen). 
Anders sieht es mit einer aktuellen Kritik aus, die sich auf die neonationalistische Instrumentalisierung jugendlicher Kraft, Spontaneität und Neuerung im Dienste eines neuen nationalen, autoritären und fremdenskeptischen bis -feindlichen Deutschlands bzw. Europas bezieht. Mit den neuen nationalistischen Jugendphänomenen europaweit gerät der Mythos Jugend als Hoffnungsträger der Moderne tendenziell in Verruf. Der hässliche Deutsche tritt nun nicht mehr als verbohrter Greis auf, sondern als smarter Jugendlicher mit Bildung und rechter Gesinnung, der als trotzige, rebellische, idealisierende und schwärmerische Figur zwar eigentlich (formal) dem Mythos Jugend entspricht, aber trotzdem nicht ins jugendbewegte Konzept der grundsätzlich positiv aufgebauten Jugendbilder passt.

Provoziert durch die neuen reaktionären, rechts- und national gesonnenen Jugendkulturen, die eigentlich so neu gar nicht sind, untersucht man jetzt die dunklen Seiten des Mythos Jugend genauer - und kommt zu desillusionierenden Ergebnissen. So wurde lange Zeit der latent immer existente reaktionäre Eigensinn des Mythos Jugend lediglich verdeckt durch die eingespielte Tradition, mit Jugend per se Aufbruch und Erneuerung zum Besseren zu verbinden. Verdächtig, aber kaum reflektiert, hätte eigentlich schon früher auffallen müssen, wie problemlos der deutsche oder italienische Faschismus den Jugendmythos der Jahrhundertwende in seine Ideologie einarbeiten konnte (Oelkers 1989).

\section{Jugendmythos und Film}

Als Fiktion und Konstruktion braucht jeder Mythos, auch der der Jugend, ein Trägermedium. Geschichten eignen sich hierzu am besten, sei es in Form von mündlich tradierten Erzählungen, in Form von Romanen oder Filmen. Erzählungen haben den Vorteil der Anschaulichkeit, der Kontextualität und der Personalisierbarkeit. In ihnen lassen sich Ideale als Figuren, Widersprüche als Ereignisse und Werte als Handlungen darstellen, und Geschichten besitzen Form und sind gleichzeitig noch formbar. D.h., jede Geschichte gibt einen Ereignisrahmen als feste Form vor, ist aber gleichzeitig in ihrer Tradierung veränderbar und interpretierbar. Alle Religionen geben sich als Mythos so eine spezifische narrative Metaphorik; man denke z.B. an die Heilige Dorothea von Cäsarea, die um 300 n. Chr. angeblich eine 
Engelserscheinung veranlasste und dafür von den Gegnern des Christentums enthauptet wurde. Die übrigen Mythen sind genauso gestrickt. Heilige, sogar Götter, werden in Geschichten eingebettet, aus denen man lernen kann; die Schicksale von Rittern, Indianern, Kriegern und Liebenden bilden mythische Kerne - und auch Jugend als konstruierte Erzählung. Schriftliche Erzählungen, z.B. Romane, erwiesen sich gegenüber mündlichen Formen der Tradierung als sehr erfolgreich, weil massenwirksam und gleichzeitig individuell rezipierbar und phantasierbar. Und es ist sicherlich kein Zufall, dass die Genese des Mythos Jugend in das Zeitalter der Romane mit seinen jugendidealisierenden Schriften von Goethe, Lafontaine, Klopstock, Herder, Hamann, Lavater oder Rousseau fällt.

Aber nicht nur reine Jugenderzählungen haben den Mythos Jugend geprägt. Der Wunsch nach Ausbruch aus der Zivilisation, ein Archetypus moderner Mythenromantik, findet im gestrandeten Robinson Crusoe von Daniel Defoe seine Projektion, ebenso wie in den Abenteuergeschichten von Karl May, der wohl wie alle Geburtshelfer mythischer Figurationen eher zufällig erfolgreich wurde. Die edlen Wilden der Welten, die ihr Autor aus Ernsthal im Erzgebirge nie vorher bereist hatte und die so viele begeisterten (auch Philosophen wie Ernst Bloch), entstanden einem wenig edlen Schöpfergeist. Karl May, der 1842 geboren wurde und in kleinbürgerlichen Verhältnissen mit vielen Geschwistern aufwuchs, schaffte zwar eine Ausbildung als Lehrer. Er wurde jedoch nicht nur wegen Betrügereien aus dem Schuldienst entfernt, sondern sass auch mehrere Jahre wegen kleinerer Vergehen im Gefängnis.

Unter diesen im wahrsten Sinne eingeschränkten Bedingungen versuchte sich der spätere Erfolgsautor zunächst mit wenig Erfolg auf literarischem Gebiet, bis er mit seinen Abenteuererzählungen auf Resonanz bei den Sehnsüchten vieler junger (und wohl auch älterer) Menschen stiess. Inspiriert durch Reiseberichte und geographische Lektüre entstanden künstlerisch eher schlichte Geschichten mit hoher Faszination. Generationen deutscher Jugendlicher durchlebten fortan in realer lebensweltlicher Enge die Heldenerlebnisse von Old Shatterhand und Kara Ben Nemsi. Der eigentliche Erfolg von Karl Mays Romanen liegt demnach in der Wechselwirkung zwischen dem schriftlich Dargebotenen und seiner phantasieanregenden Rezeption durch das jugendliche Lesepublikum. Die eigentlichen 
Geschichten von Karl May entstehen als kollektive Phantasmen in den Köpfen und entfalten hier ihre mythische Kraft.

Filme können diese narrative und mythengenerierende Tradition der Literatur direkt aufgreifen. Sie bieten Projektionsflächen im eigentlichen, aber auch im übertragenen Sinn und können dem Mythos Jugend eine akustische und optische Form geben. Auch Filme treten als Massenmedien auf, nehmen während der Rezeption individuell gefangen und finden trotz ihrer konkreten Bildersprache in Phantasien, eigentlich im Kopfkino, ihre Fortsetzung.

Und wie bei Karl May brauchen die Jugendmythen in Filmen keine künstlerische Qualität oder aufwendige Inhalte. Vielmehr kommt es darauf an, dass sich Jugendliche mit den Filmen identifizieren und sie als Sprungbrett in alternative Phantasiewelten nutzen können. Dabei spielt die rekursive Wechselwirkung zwischen dem Bedürfnis nach einem Mythos und mythischen Angeboten eine wichtige Rolle. Filmerzählungen werden dann zu Filmmythen, wenn sie einem nur in Ahnungen gegenwärtigen und in Bruchstücken reflektierten jugendlichen Lebensgefühl Gestalt geben können, wenn sie einer bislang ungestalteten Sehnsucht eine konkrete Form geben und der so entstehende Mythos dann die Züge eines Wesenskerns von Wirklichkeit annimmt. Besonders der letzte Schritt ist wichtig, macht doch erst die Verwandlung der Konstruktion (z.B. der Erzählung), des Erfundenen in ein kollektiv geteiltes Wissen über eine Erzählung (nun als gefestigte Realität) den Mythos zum Mythos. Dieser Schritt ist bei Filmen oder Romanen gleich. Stendhal beschreibt das sehr schön in Rot und Schwarz. Dieser Roman von 1830 trägt den Untertitel Chronik des 19. Jahrhunderts und beschreibt mit deutlich autobiographischen Zügen, wie der Held der Geschichte, der junge Julien Sorel, als Sohn eines Zimmermanns die Provinz verlässt und die hohe Gesellschaft in den Städten Frankreichs kennenlernt. Er muss allerdings erst mühsam lernen, sich in dieser ihm fremden Welt zurecht zu finden, weil er die Geschichten nicht kennt, die hier Massstab und Orientierung geben. Und diese Geschichten sind die eleganten Romane der Zeit, die in Paris und anderen Grossstädten verschlungen werden, in der Provinz aber unbekannt oder verpönt sind. Während also die anderen jungen Gecken durch das Studium der neuesten Romane genau wissen, welche Konversation in einem Salon gerade en 
vogue ist, wann man erstaunt oder gelangweilt tut und wie man einen Liebesbrief schreibt, durchlebt Julien dieselben Irritationen, die Kant über sich als 18-Jähriger schreibt:

«Lächerliche und rührende Erinnerungen: der erste Salon, in dem man sich mit achtzehn Jahren zeigt, allein und ohne Beistand! Der Blick einer Frau genügte, um mich einzuschüchtern. Je mehr ich gefallen wollte, desto linkischer wurde ich. Vor allem machte ich mir falsche Vorstellungen, oder schwärmte ohne Grund, oder sah in einem Mann einen Feind, weil er mich streng angeblickt hatte. Und doch, wie schön war damals ein Tag!» (aus: Stendhal 2004)

Sicherheit - und manchmal eben auch Langeweile - schafft dagegen der Roman; heute vornehmlich als Film und früher als Literatur werden über Medien die Stereotype verbreitet, die kollektive Orientierung und jedem ersten eigenen Erlebnis eine Ahnung von Déjà-vu geben. Niklas Luhmann gründet auf Romanen, von denen Stendal schreibt, sie seien wie Spiegel, die man eine Strasse entlang trägt, seine soziologische Untersuchung des modernen Liebescodes: Liebe als Passion (Luhmann 1982). Filmmythen, und das ist unsere These, sind ebensolche Spiegel, in denen sich Jugendliche heute wiedererkennen können und auf die sie ihr wahres Leben projizieren können. Zwar droht damit der Erfahrungswelt gegenüber den zumeist spektakulären Medienvorbildern immer die Gefahr, trist und langweilig zu sein, aber der Mythos Jugend in den Filmen lässt sich immerhin symbolisch über Kopierversuche (Moden, Gesten, Mentalitäten etc.) in den Alltag übertragen.

Geht man nun auf die Suche nach Jugendmythen im Film, so können aus denjenigen Filmen, die zum Mythos Jugend beigetragen haben, einige Charakteristika herausgearbeitet werden. Zunächst einmal bekommt der Mythos Jugend im Film Präsenz und Anschaulichkeit über die mitwirkenden Darstellerinnen und Darsteller, die Charaktere. Weiterhin sind es die Film-Geschichten, die dem Mythos Jugend eine konkrete Metaphorik verleihen können. Als Charaktere führen wir im Folgenden das etwas museale Beispiel James Dean an, der durch seinen Tod als mythische Jugendfigur weiterexistieren konnte. Die Geschichte inszeniert die (amerikanische) Jugendphase (der 6oer Jahre) in einem verklärten Rückblick als ungebundene Übergangssituation ins spätere Erwachsenenalter. 


\section{Filmmythen: James Dean - Jugend als Rebellion}

Der Schauspieler James Dean wurde 1931 in Marion, Indiana, mit dem bürgerlichen Namen James Byron Dean geboren und entwickelte sich später $\mathrm{zu}\langle\mathrm{dem}\rangle$ Jugendidol der 50er und 6oer Jahre in den USA und auch international. Seine Biographie nimmt sich zuerst einmal recht unspektakulär aus, aber der Mythos James Dean ist ja auch nicht an die reale Person des Schauspielers gekoppelt, sondern an seine Filmimagination. James Dean studierte in Los Angeles Rechts- und Theaterwissenschaften und machte erste schauspielerische Erfahrungen an einem Studententheater. Relativ konsequent entwickelte sich dann sein Weg als Schauspieler. Sein Ausbildungsweg verlief klassisch und weist eigentlich keine Spuren jugendlichen Rebellentums auf. Von der Schauspielschule der University of California kam er auf das berühmte New Yorker Actors Studio, eine Schauspielschule von Lee Strasberg geführt, die auch schon Marlon Brando besucht hatte. Als Schauspielanfänger musste sich der junge James Dean wie viele andere Kolleginnen und Kollegen seinen Lebensunterhalt durch Auftritte in Werbespots oder in unbedeutenden Fernsehfilmen verdienen, die späteren prominenten Auftritte waren zu Beginn noch nicht in Sicht. Doch schon bald machte James Dean Karriere, und mit seinem beruflichen Erfolg entstand der Mythos vom trotzigen Jugendlichen, der eigentlich nie Erfolg hat, auch nicht haben will, sich stattdessen in Probleme manövriert, diese aber auch mit einer trotzigen Antihaltung zu meistern versucht. Der Weg zum Erfolg des Schauspielers führte wieder über das Actors Studio; am Broadway wurde man auf den 21-jährigen 1952 aufmerksam, der im Bühnenstück 〈The Immoralist) mitspielte, was ihm mehrere Auszeichnungen einbrachte. Sein erster grosser Spielfilm, (Jenseits von Eden), aufgeführt zuerst 1955, machte dann Filmgeschichte. Die Story wies dem nun 24-jährigen James Dean in der Hauptrolle die Figur eines Jugendlichen zu, der sich gegen die kleinbürgerliche Enge seiner Familie auflehnt. Cal Trask, so der Name des Jugendlichen im Film, repräsentiert eine durchaus problematische Figur in problematischen Verhältnissen, und schien in der brillanten Verkörperung durch den Schauspieler den Nerv vieler Jugendlicher getroffen zu haben. So erhielt James Dean für diese Rolle nicht nur einen Oscar, sondern wurde auch zur Identifikationsfigur. Auch im Jahr 1955 erscheint ein weiterer Film mit James Dean, Nicolas Rays «Denn sie wissen 
nicht, was sie tun>. Dieser Film begründete schlussendlich den Jugendmythos James Dean und auch den Mythos des rebellierenden und coolen amerikanischen Jugendlichen (eigentlich: Jungen), der in einer Jugendwelt erster Liebesaffären, der Jungenfreundschaften, Musik und Peerkonflikte aufwächst, mit Autos, Zigaretten und Alkohol erste Flaggensignale des Erwachsenseins setzt, sich gegen Erwachsene, Polizisten und Gangs behaupten muss und langsam in die eigentlich ungeliebte Rolle des Erwachsenen hineinschliddert. Probleme müssen in dieser Jugendwelt allein ausgetragen werden, ähnlich wie Initiationsaufgaben oder Mutproben, allerdings gelingt das nicht immer, sodass auch Scheitern (heute würde man sagen: Identitätsprobleme und Devianz) ein Teil des Jugendmythos wird. Jim Stark, so heisst die von James Dean verkörperte Figur, kommt in «Denn sie wissen nicht, was sie tun〉 als (new kid in the town〉 eingesessenen Jugendbanden ins Gehege und gibt damit einem speziellen jugendlichen Archetypus Gestalt, der (ähnlich wie ein Westernheld) in Coolness und Einsamkeit seinen Mann stehen muss und diese Herausforderung auch annimmt. Berühmt und symbolträchtig für den Film wie auch den darüber gezeichneten Jugendmythos ist die Szene des Autorennens auf einen Abgrund zu. Es geht darum, im Zweikampf auf einen Steilhang zuzurasen und sich im letzten Moment aus dem Auto rollen zu lassen, bevor der Wagen über die Abbruchkante fliegt. James Dean kann sich aus dem Auto fallen lassen, er überlebt (und verliert); sein Kontrahent hingegen verhakt sich am Türgriff und stirbt im Wagen (gewinnt deswegen aber). Mit anderen Worten: Die Anti-Aktionen dieser Filmjugendlichen der 5oer Jahre gegen die (Erwachsenen-)Welt brauchen anscheinend noch keine dritte Ratio (etwa Protest gegen die etablierte Politik, Klimawandel oder soziale Ungleichheit), sondern genügten sich selbst. Und die Figur des Rebellen à la James Dean konnte eine bis dahin in den USA und noch stärker in Deutschland überdisziplinierte Jugend allein dadurch faszinieren, dass sie sich trotzig und 〈ohne zu wissen, was man genau tut〉, gegen die autoritäre Erwachsenenwelt anstemmte. 


\section{Literatur}

Barthes, Roland. 2010. Mythen des Alltags. Berlin: Suhrkamp.

Frank, Manfred. 1989. Kaltes Herz. Unendliche Fahrt. Neue Mythologie. Motiv-Untersuchungen zur Pathogenese der Moderne. Frankfurt a. M.: Suhrkamp.

Luhmann, Niklas. 1982. Liebe als Passion. Zur Codierung von Intimität. Frankfurt a. M.: Suhrkamp.

Nietzsche, Friedrich. 1969. Werke in drei Bänden. Herausgegeben von Karl Schlechter. München.

Oelkers, Jürgen. 1989. Reformpädagogik - Eine kritische Dogmengeschichte. Weinheim/München: Beltz.

Sander, Uwe. 2000. «100 Jahre Jugend in Deutschland». DVJJ-Journal. Zeitschrift für Jugendkriminalrecht und Jugendhilfe 2/200, 167-73.

Stendahl, Marie-Henri Beyle. 2004. Rot und Schwarz. München: Hanser. 